\title{
Nonnoetherian geometry
}

\author{
Charlie Beil \\ Heilbronn Institute for Mathematical Research \\ School of Mathematics, Howard House \\ The University of Bristol \\ Bristol, BS8 1SN, UK \\ charlie.beil@bristol.ac.uk \\ Received 8 April 2015 \\ Accepted 30 October 2015 \\ Published 6 January 2016 \\ Communicated by P. Smith
}

\begin{abstract}
We introduce a theory of geometry for nonnoetherian commutative algebras with finite Krull dimension. In particular, we establish new notions of normalization and height: depiction (a special noetherian overring) and geometric codimension. The resulting geometries are algebraic varieties with positive-dimensional points, and are thus inherently nonlocal. These notions also give rise to new equivalent characterizations of noetherianity that are primarily geometric. We then consider an application to quiver algebras whose simple modules of maximal dimension are one dimensional at each vertex. We show that the vertex corner rings of $A$ are all isomorphic if and only if $A$ is noetherian, if and only if the center $Z$ of $A$ is noetherian, if and only if $A$ is a finitely generated $Z$-module. Furthermore, we show that $Z$ is depicted by a commutative algebra generated by the cycles in its quiver. We conclude with an example of a quiver algebra where projective dimension and geometric codimension, rather than height, coincide.
\end{abstract}

Keywords: Nonnoetherian rings; foundations of algebraic geometry; noncommutative algebraic geometry.

Mathematics Subject Classification: 13C15, 14A20

\section{Introduction}

The purpose of this paper is to introduce a new framework for understanding the geometry of nonnoetherian algebras with finite Krull dimension. The underlying idea we propose is that nonnoetherian geometry should be the geometry of nonlocal algebraic varieties.

This is an Open Access article published by World Scientific Publishing Company. It is distributed under the terms of the Creative Commons Attribution 4.0 (CC-BY) License. Further distribution of this work is permitted, provided the original work is properly cited. 
We first motivate our study of nonnoetherian geometry in the following applications.

(1) Noncancellative dimer algebras. A dimer algebra is a type of quiver algebra whose quiver embeds in a torus, with homotopy-like relations. These noncommutative algebras encode certain gauge theories in string theory. Dimer algebras with the cancellation property are Calabi-Yau algebras, and their centers are threedimensional Gorenstein singularities. Noncancellative dimer algebras, on the other hand, are not Calabi-Yau, and their centers are nonnoetherian. In contrast to their cancellative counterparts, very little is known about these algebras, despite the fact that almost all dimer algebras are noncancellative. In [3], we use the tools developed here to show that their centers are also three-dimensional singularities, but with the strange property that they contain positive-dimensional "smearedout" points. Furthermore, in [4] we describe how this nonlocal geometry is reflected in the homology of their noncommutative residue fields.

(2) Quantum entanglement and nonlocality. In quantum physics, two entangled particles can effect each instantaneously no matter how great their spatial separation. In $[5,6]$, we introduce a toy model where such entangled states are supported on nonnoetherian singularities of spacetime. Furthermore, we show that the noncommutative blowup of the singular support of an entangled Bell state leads to the notion that the nonlocal commutative spacetime that we observe emerges from an underlying local noncommutative spacetime [5, Theorem $\mathrm{C}]$.

(3) Nonnoetherian geometry. In algebraic geometry, one may ask what is the geometry of a nonnoetherian algebra $R$ ? The usual answer is the affine scheme Spec $R$ whose global sections are $R$. However, schemes are abstract objects, and we would like an answer to this question that we can visualize. We aim to give such an answer here.

We now outline our main results.

In Sec. 2 we introduce the idea of a nonlocal algebraic variety. To illustrate this notion, consider the algebra $S=k[x, y]$ and its nonnoetherian subalgebra

$$
R=k\left[x, x y, x y^{2}, \ldots\right]=k+x S .
$$

The maximal ideal spectrum $\operatorname{Max} R$ of $R$ may be viewed as two-dimensional affine space $\mathbb{A}_{k}^{2}=\operatorname{Max} S$ with the line

$$
\mathcal{Z}(x)=\{x=0\} \subset \mathbb{A}_{k}^{2}
$$

identified as a single closed point. From this perspective, $\mathcal{Z}(x)$ is a one-dimensional "smeared-out" point of $R$, and therefore $\operatorname{Max} R$ is nonlocal.

More generally, let $S$ be an integral domain and a finitely generated $k$-algebra, and let $R$ be a possibly nonnoetherian subalgebra of $S$. In order to capture the locus where $\operatorname{Max} R$ "looks like" the variety $\operatorname{Max} S$, we introduce the open subset

$$
U_{S / R}:=\left\{\mathfrak{n} \in \operatorname{Max} S \mid R_{\mathfrak{n} \cap R}=S_{\mathfrak{n}}\right\}
$$


Theorem A (Theorem 2.5). Suppose $U_{S / R} \neq \emptyset$. Then $\operatorname{Max} S$ and $\operatorname{Max} R$ are isomorphic on open dense subsets, and thus birationally equivalent. Furthermore, the Krull dimensions of $R$ and $S$ are equal.

In example (1), $U_{S / R}$ is the complement to the subvariety $\mathcal{Z}(x)$. We generalize this example by showing that if $R$ is generated by a subalgebra of $S$ and an ideal $I \subset S$, then $U_{S / R}$ contains the complement to the subvariety $\mathcal{Z}(I)$ in $\operatorname{Max} S$. In addition, if $I$ is a nonmaximal radical ideal of $S$ and $R=k+I$, then $U_{S / R}=\mathcal{Z}(I)^{c}$ (Proposition 2.8).

To formalize these notions, we introduce the following definitions.

\section{Definition B (Definition 3.1).}

- A finitely generated $k$-algebra $S$ is a depiction of a subalgebra $R \subseteq S$ if

(i) the morphism $\iota_{S}: \operatorname{Spec} S \rightarrow \operatorname{Spec} R, \mathfrak{q} \mapsto \mathfrak{q} \cap R$, is surjective,

(ii) for each $\mathfrak{n} \in \operatorname{Max} S, R_{\mathfrak{n} \cap R}$ is noetherian iff $R_{\mathfrak{n} \cap R}=S_{\mathfrak{n}}$, and

(iii) $U_{S / R} \neq \emptyset$.

- The geometric codimension or geometric height of $\mathfrak{p} \in \operatorname{Spec} R$ is the infimum

$$
\operatorname{ght}(\mathfrak{p}):=\inf \left\{\operatorname{ht}(\mathfrak{q}) \mid \mathfrak{q} \in \iota_{S}^{-1}(\mathfrak{p}), S \text { a depiction of } R\right\}
$$

The geometric dimension of $\mathfrak{p}$ is the difference

$$
\operatorname{gdim} \mathfrak{p}:=\operatorname{dim} R-\operatorname{ght}(\mathfrak{p}) .
$$

In example (1), $R$ is depicted by $S$, and the geometric dimension of the closed point $\mathcal{Z}(x)$ of $R$ is 1 (Example 3.11 ). The following theorem describes the fundamental geometry of nonnoetherian algebras with finite krull dimension.

Theorem C (Theorems 3.8, 3.12, 3.13, and Proposition 3.16). Suppose $R$ admits a depiction $S$ and let $\mathfrak{p} \in \operatorname{Spec} R$. Then

$$
\operatorname{ght}(\mathfrak{p}) \leq \operatorname{ht}(\mathfrak{p}),
$$

with equality if there is some $\mathfrak{q} \in \operatorname{Spec} S$ for which $\mathfrak{q} \cap R=\mathfrak{p}$ and $\mathcal{Z}(\mathfrak{q}) \cap U_{S / R} \neq \emptyset$.

Furthermore, the following conditions are equivalent:

(1) $R$ is noetherian.

(2) $U_{S / R}=\operatorname{Max} S$.

(3) $R=S$.

In particular, if $R$ is noetherian, then its only depiction is itself.

Finally, if $\operatorname{Max} R$ contains a closed point of positive geometric dimension, then $R$ is nonnoetherian. Conversely, if $R$ is an isolated nonnoetherian singularity, then $\operatorname{Max} R$ contains a closed point of positive geometric dimension.

Consequently, if $I$ is a radical ideal of a finitely generated $k$-algebra $S$, then the $\operatorname{ring} R=k+I$ will be nonnoetherian if and only if $\operatorname{dim} \mathcal{Z}(I) \geq 1$ (Corollary 3.14). 
We conclude the section by showing that depictions which are minimal with respect to inclusion do not exist in general, and maximal depictions are not unique in general (Proposition 3.19).

In Sec. 4, we study nonlocality in the context of noncommutative algebraic geometry. Let $A$ be a finitely generated noncommutative $k$-algebra with center $Z$. We consider algebras with the following particularly nice matrix ring structure.

Definition 1.1. An impression of $A$ is a finitely generated commutative $k$-algebra $B$ and an algebra monomorphism $\tau: A \hookrightarrow M_{d}(B)$ such that (i) for each $\mathfrak{b}$ in some open dense subset of $\operatorname{Max} B$, the composition with the evaluation map

$$
A \stackrel{\tau}{\rightarrow} M_{d}(B) \stackrel{\epsilon_{\mathfrak{b}}}{\rightarrow} M_{d}(B / \mathfrak{b})
$$

is surjective, and (ii) the morphism $\operatorname{Max} B \rightarrow \operatorname{Max} \tau(Z), \mathfrak{b} \mapsto \mathfrak{b} 1_{d} \cap \tau(Z)$, is surjective [1, Definition and Lemma 2.1].

An impression is useful in part because it determines the center $Z$ of $A[1$, Lemma 2.1]. Furthermore, if $A$ is a finitely generated $Z$-module, then $\tau$ determines all simple $A$-module isoclasses of maximal $k$-dimension [1, Proposition 2.5].

Denote by $E_{j i} \in M_{d}(k)$ the matrix with a 1 in the $(j i)$ th slot and zeros elsewhere. Let $A=k Q / I$ be a quiver algebra with vertex set $Q_{0}=\{1, \ldots, d\}$, and suppose $A$ admits an impression $\tau: A \hookrightarrow M_{d}(B)$ such that $\tau\left(e_{i}\right)=E_{i i}$ for each $i \in Q_{0}$. For $p \in e_{j} A e_{i}$, denote by $\bar{\tau}(p) \in B$ the single nonzero entry of the matrix $\tau(p)$; whence

$$
\tau(p)=\bar{\tau}(p) E_{j i} .
$$

For each $i, j \in Q_{0}, \bar{\tau}$ defines a $k$-linear map $\bar{\tau}: e_{j} A e_{i} \rightarrow B$. Set

$$
\begin{aligned}
R & :=k\left[\cap_{i \in Q_{0}} \bar{\tau}\left(e_{i} A e_{i}\right)\right] \subseteq B, \\
S & :=k\left[\cup_{i \in Q_{0}} \bar{\tau}\left(e_{i} A e_{i}\right)\right] \subseteq B .
\end{aligned}
$$

Theorem D (Theorem 4.1). Suppose $\tau: A \hookrightarrow M_{\left|Q_{0}\right|}(B)$ is an impression of $A$ with $B$ an integral domain and $\tau\left(e_{i}\right)=E_{i i}$ for each $i \in Q_{0}$. Then $U_{S / R} \neq \emptyset$. Furthermore, if $\left\{\mathfrak{n} \in \operatorname{Max} S \mid R_{\mathfrak{n} \cap R}\right.$ is noetherian $\} \subseteq U_{S / R}$, then

(1) The center $Z$ of $A$ is isomorphic to $R$ and is depicted by $S$.

(2) The statements

(a) $R=S$;

(b) $A$ is a finitely generated $Z$-module;

(c) $Z$ is noetherian;

(d) A is noetherian;

satisfy the equivalences

$$
(\mathrm{a}) \Longleftrightarrow(\mathrm{b}) \Longleftrightarrow(\mathrm{c}) \rightleftharpoons(\mathrm{d})
$$

where $(\star)$ holds if the $\bar{\tau}$-image of a path is a monomial in $B$ and $I$ is generated by binomials in the paths of $Q$. 
Again consider the nonnoetherian algebra $R=k+x S$ in example (1). In Example 4.3 and Proposition 4.4, we study the endomorphism ring

$$
A=\operatorname{End}_{R}(R \oplus x S)
$$

of the reflexive $R$-module $R \oplus x S$. This algebra may be viewed as the noncommutative blowup of $R$ at the isolated singular point $x S$ of $\operatorname{Max} R[7, \operatorname{Sec} . \mathrm{R}]$. Furthermore, $A$ admits an impression $\tau: A \hookrightarrow M_{2}(S)$ satisfying

$$
R=k+x S=k\left[\cap_{i \in Q_{0}} \bar{\tau}\left(e_{i} A e_{i}\right)\right] \quad \text { and } \quad S=k[x, y]=k\left[\cup_{i \in Q_{0}} \bar{\tau}\left(e_{i} A e_{i}\right)\right]
$$

By Theorem D, $A$ is nonnoetherian, has center $R$, and is an infinitely generated $R$-module. We show that the projective dimension and geometric codimension of its vertex simple modules $V_{i}$ coincide:

$$
\operatorname{pd}_{A}\left(V_{i}\right)=\operatorname{ght}\left(\operatorname{ann}_{e_{i} A e_{i}}\left(V_{i}\right)\right) .
$$

This example suggests that the notion of geometric dimension is, in a suitable sense, a natural definition.

Notation. We will denote by $\operatorname{dim} R$ the Krull dimension of $R$; by Frac $R$ the $\operatorname{ring}$ of fractions of $R$; by $\operatorname{Max} R$ the set of maximal ideals of $R$; and by $\operatorname{Spec} R$ either the set of prime ideals of $R$ or the affine $k$-scheme with global sections $R$. For $\mathfrak{a} \subset R$ we will denote by $\mathcal{Z}(\mathfrak{a})$ either the closed set $\{\mathfrak{m} \in \operatorname{Max} R \mid \mathfrak{m} \supseteq \mathfrak{a}\}$ of $\operatorname{Max} R$ or the closed set $\{\mathfrak{p} \in \operatorname{Spec} R \mid \mathfrak{p} \supseteq \mathfrak{a}\}$ of $\operatorname{Spec} R$, depending on the context. For a subset $Y$ of $\operatorname{Max} S$, set $Y^{c}:=\operatorname{Max} S \backslash Y$.

In Sec. 4 we will denote by $Q=\left(Q_{0}, Q_{1}, \mathrm{t}, \mathrm{h}\right)$ a quiver with vertex set $Q_{0}$, arrow set $Q_{1}$, and head and tail maps h, t $: Q_{1} \rightarrow Q_{0}$. We will denote by $k Q$ the path algebra of $Q$, and by $e_{i}$ the idempotent corresponding to the vertex $i \in Q_{0}$. Multiplication of paths is read right to left, following the composition of maps. By module we mean left module. By infinitely generated $R$-module, we mean a module that is not finitely generated over $R$.

\section{Nonnoetherian Geometry as Nonlocal Geometry}

Throughout $k$ is an algebraically closed field; $S$ is an integral domain and a noetherian $k$-algebra; and $R$ is a (possibly nonnoetherian) subalgebra of $S$. We begin with the following well-known lemma.

Lemma 2.1. If $\mathfrak{q} \in \operatorname{Spec} S$, then $\mathfrak{q} \cap R \in \operatorname{Spec} R$. Furthermore, if $S$ is a finitely generated $k$-algebra and $\mathfrak{n} \in \operatorname{Max} S$, then $\mathfrak{n} \cap R \in \operatorname{Max} R$.

Proof. We show the second statement. Suppose $S$ is a finitely generated $k$-algebra and let $\mathfrak{n} \in \operatorname{Max} S$. Then $S / \mathfrak{n} \cong k$ since $S$ is finitely generated over the algebraically 
closed field $k{ }^{\text {a }}$ Thus the composition $\psi: R \hookrightarrow S \rightarrow S / \mathfrak{n}$ is surjective since $1_{S} \in R$. Whence $R / \operatorname{ker} \psi \cong k$. Therefore $\operatorname{ker} \psi=\mathfrak{n} \cap R$ is a maximal ideal of $R$.

Consider the morphisms

$$
\begin{array}{rlrl}
\iota: \operatorname{Spec} S & \rightarrow \operatorname{Spec} R \quad \text { and } \quad \kappa: \operatorname{Max} S \rightarrow \operatorname{Spec} R, \\
\mathfrak{q} & \mapsto \mathfrak{q} \cap R & \mathfrak{n} \mapsto \mathfrak{n} \cap R .
\end{array}
$$

Let $\mathfrak{q} \in \operatorname{Spec} S$ and set $\mathfrak{p}=\iota(\mathfrak{q})=\mathfrak{q} \cap R$. Then $R \backslash \mathfrak{p} \subseteq S \backslash \mathfrak{q}$. Whence

$$
R_{\iota(\mathfrak{q})}=R_{\mathfrak{p}} \subseteq S_{\mathfrak{q}} .
$$

Thus the embedding $R \hookrightarrow S$ induces the morphism of schemes

$$
\iota:\left(\operatorname{Spec} S, \mathcal{O}_{\mathrm{Spec} S}\right) \rightarrow\left(\operatorname{Spec} R, \mathcal{O}_{\mathrm{Spec} R}\right) .
$$

To aid our analysis of $R$, we introduce the following subsets of Max $S$ and $\operatorname{Spec} S$ :

$$
\begin{aligned}
U_{S / R} & :=\left\{\mathfrak{n} \in \operatorname{Max} S \mid R_{\mathfrak{n} \cap R}=S_{\mathfrak{n}}\right\}, \\
\tilde{U}_{S / R} & :=\left\{\mathfrak{q} \in \operatorname{Spec} S \mid R_{\mathfrak{q} \cap R}=S_{\mathfrak{q}}\right\}, \\
U_{S / R}^{*} & :=\left\{\mathfrak{n} \in \operatorname{Max} S \mid R_{\mathfrak{n} \cap R} \text { is noetherian }\right\}, \\
W_{S / R} & :=\{\mathfrak{n} \in \operatorname{Max} S \mid \sqrt{(\mathfrak{n} \cap R) S}=\mathfrak{n}\} .
\end{aligned}
$$

These subsets will play a central role throughout this paper. Furthermore, $U_{S / R}$ will play a central role in its sequels $[3,2]$ in the context of dimer algebras. If $R$ and $S$ are fixed, then we will often omit the subscript $S / R$.

Lemma 2.2. Suppose $\mathfrak{p} \in \operatorname{Spec} R$ and $\mathfrak{q} \in \iota^{-1}(\mathfrak{p})$. If $\mathcal{Z}(\mathfrak{q}) \cap U \neq \emptyset$, then $R_{\mathfrak{p}}=S_{\mathfrak{q}}$.

Proof. Let $\mathfrak{n} \in \mathcal{Z}(\mathfrak{q}) \cap U$ and set $\mathfrak{m}=\mathfrak{n} \cap R$. Then $R_{\mathfrak{m}}=S_{\mathfrak{n}}$. Furthermore, $R_{\mathfrak{p}} \subseteq S_{\mathfrak{q}}$ by (2). Thus, since $\mathfrak{p} \subseteq \mathfrak{q}$,

$$
S_{\mathfrak{q}}=\left(S_{\mathfrak{n}}\right)_{\mathfrak{q}} \subseteq\left(S_{\mathfrak{n}}\right)_{\mathfrak{p}}=\left(R_{\mathfrak{m}}\right)_{\mathfrak{p}}=R_{\mathfrak{p}} \subseteq S_{\mathfrak{q}}
$$

Therefore $R_{\mathfrak{p}}=S_{\mathfrak{q}}$.

Lemma 2.3. Suppose $\mathfrak{q}, \mathfrak{q}^{\prime} \in \operatorname{Spec} S$ satisfy

$$
\mathfrak{q} \cap R=\mathfrak{q}^{\prime} \cap R \quad \text { and } \quad \mathfrak{q} \subseteq \mathfrak{q}^{\prime} .
$$

If $\mathcal{Z}(\mathfrak{q}) \cap U \neq \emptyset$ or $\mathcal{Z}\left(\mathfrak{q}^{\prime}\right) \cap U \neq \emptyset$, then $\mathfrak{q}=\mathfrak{q}^{\prime}$.

Proof. We claim that $S_{\mathfrak{q}}=S_{\mathfrak{q}^{\prime}}$, and consequently $\mathfrak{q}=\mathfrak{q}^{\prime}$ since $S_{\mathfrak{q}}$ has a unique maximal ideal.

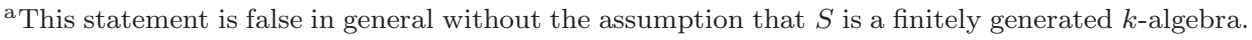
Indeed, let $S$ be $\mathbb{C}$, let $k$ be the algebraic closure of $\mathbb{Q}$, and let $\mathfrak{n}$ be the maximal ideal 0 of $\mathbb{C}$. Then $S / \mathfrak{n} \neq k$.
} 
Indeed, suppose $\mathcal{Z}(\mathfrak{q}) \cap U \neq \emptyset$. Then by Lemma 2.2,

$$
S_{\mathfrak{q}^{\prime}} \subseteq S_{\mathfrak{q}}=R_{\mathfrak{q} \cap R}=R_{\mathfrak{q}^{\prime} \cap R} \subseteq S_{\mathfrak{q}^{\prime}} .
$$

Therefore $S_{\mathfrak{q}}=S_{\mathfrak{q}^{\prime}}$.

So suppose $\mathcal{Z}\left(\mathfrak{q}^{\prime}\right) \cap U \neq \emptyset$. Since $\mathfrak{q} \subseteq \mathfrak{q}^{\prime}$, we have

$$
\mathcal{Z}\left(\mathfrak{q}^{\prime}\right) \subseteq \mathcal{Z}(\mathfrak{q})
$$

Whence $\mathcal{Z}(\mathfrak{q}) \cap U \neq \emptyset$, which was the previous case.

In the remainder of this section we assume that $S$ is a finitely generated $k$-algebra, unless stated otherwise. Recall that $S$ is an overring of a domain $R$ if $R \subseteq S \subseteq \operatorname{Frac} R$.

Proposition 2.4. Suppose $U$ is nonempty. Then

(1) $S$ is an overring of $R$; and

(2) $U$ is an open dense subset of $\operatorname{Max} S$.

In particular, the function fields of $\operatorname{Spec} S$ and $\operatorname{Spec} R$ coincide.

Proof. (1) Suppose $\mathfrak{n} \in U:=U_{S / R}$. Since $S$ is an integral domain, we have

$$
\operatorname{Frac} S=\operatorname{Frac}\left(S_{\mathfrak{n}}\right)=\operatorname{Frac}\left(R_{\mathfrak{n} \cap R}\right)=\operatorname{Frac} R .
$$

(2) We first claim that $U$ coincides with the locus

$$
U^{\prime}:=\left\{\mathfrak{n} \in \operatorname{Max} S \mid S \subseteq R_{\mathfrak{n} \cap R}\right\} .
$$

Indeed, let $\mathfrak{n} \in U$. Then $S_{\mathfrak{n}}=R_{\mathfrak{n} \cap R}$. Whence $S \subseteq R_{\mathfrak{n} \cap R}$. Thus $U \subseteq U^{\prime}$.

Conversely, suppose $\mathfrak{n} \in U^{\prime}$. Then

$$
S_{\mathfrak{n}}=S(S \backslash \mathfrak{n})^{-1} \subseteq R_{\mathfrak{n} \cap R}\left(R_{\mathfrak{n} \cap R} \backslash\left(\mathfrak{n} \cap R_{\mathfrak{n} \cap R}\right)\right)^{-1}=R_{\mathfrak{n} \cap R}=S_{\mathfrak{n}} .
$$

Whence $R_{\mathfrak{n} \cap R}=S_{\mathfrak{n}}$. Thus $U \supseteq U^{\prime}$. Therefore $U=U^{\prime}$, proving our claim.

Suppose that $U=U^{\prime}$ is nonempty. By assumption, $S$ is a finitely generated $k$-algebra. Thus there is a finite set $T:=\left\{s_{1}, \ldots, s_{\ell}\right\} \subset S$, minimal with respect to inclusion, such that

$$
S=R\left[s_{1}, \ldots, s_{\ell}\right]
$$

By claim (1), there are elements $r_{1}, \ldots, r_{\ell} \in R$ such that $r_{i} s_{i} \in R$. Whence $s_{i} \in$ $R\left[r_{i}^{-1}\right]$. Therefore

$$
S \subseteq R\left[r_{1}^{-1}, \ldots, r_{\ell}^{-1}\right]
$$

Consider the open set

$$
U_{T}^{\prime \prime}:=\bigcap_{1 \leq i \leq \ell} D\left(r_{i}\right),
$$


where $D\left(r_{i}\right)=\left\{\mathfrak{n} \in \operatorname{Max} S \mid \mathfrak{n} \not \supset r_{i}\right\}$ is the principal open set where $r_{i}$ does not vanish. Then

$$
U_{T}^{\prime \prime} \subseteq U^{\prime}
$$

Furthermore, $U^{\prime}$ is the union over all such minimal sets $T \subset S$,

$$
U^{\prime}=\bigcup_{T} U_{T}^{\prime \prime}
$$

In particular, $U^{\prime}$ is open. Thus $U^{\prime}$ is dense since $S$ is an integral domain and $U^{\prime}$ is nonempty. Therefore $U=U^{\prime}$ is open dense.

The morphism $\iota: \operatorname{Spec} S \rightarrow \operatorname{Spec} R$ is injective on the subset $\tilde{U} \subseteq \operatorname{Spec} S$ defined in (3). Indeed, if $\mathfrak{q} \cap R=\mathfrak{q}^{\prime} \cap R$ and $\mathfrak{q}, \mathfrak{q}^{\prime} \in \tilde{U}$, then

$$
S_{\mathfrak{q}}=R_{\mathfrak{q} \cap R}=R_{\mathfrak{q}^{\prime} \cap R}=S_{\mathfrak{q}^{\prime}} .
$$

Whence $\mathfrak{q}=\mathfrak{q}^{\prime}$. This fact is generalized in the following theorem.

Furthermore, we will find that the locus $W \subset \operatorname{Max} S$ is similar in spirit to the Azumaya locus of $A$ when $A$ is a noncommutative algebra, module-finite over its center $Z$. ${ }^{\mathrm{b}}$

Theorem 2.5. Let $R$ be a subalgebra of $S$.

(1) If $R_{\mathfrak{q} \cap R}=S_{\mathfrak{q}}$, then

$$
\iota^{-1} \iota(\mathfrak{q})=\{\mathfrak{q}\}
$$

In particular, this holds if $\mathcal{Z}(\mathfrak{q}) \cap U_{S / R} \neq \emptyset$.

(2) The locus $W_{S / R}$ is the subset of all $\mathfrak{n} \in \operatorname{Max} S$ for which

$$
\kappa^{-1} \kappa(\mathfrak{n})=\{\mathfrak{n}\}
$$

In particular, $U_{S / R} \subseteq W_{S / R}$.

(3) If $U_{S / R} \neq \emptyset$, then $\operatorname{Max} S$ and $\operatorname{Max} R$ are isomorphic on open dense subsets, and thus birationally equivalent.

(4) If $U_{S / R} \neq \emptyset$, then the Krull dimensions of $R$ and $S$ coincide,

$$
\operatorname{dim} R=\operatorname{dim} S
$$

Proof. (1.i) Let $\mathfrak{q} \in \tilde{U}_{S / R}$, and suppose $\mathfrak{q}^{\prime} \in \iota^{-1} \iota(\mathfrak{q})$. We want to show that $\mathfrak{q}^{\prime}=\mathfrak{q}$. Set $\mathfrak{p}:=\mathfrak{q} \cap R=\mathfrak{q}^{\prime} \cap R$. Then

$$
\mathfrak{p}_{\mathfrak{p}}=(\mathfrak{q} \cap R) R_{\mathfrak{p}} \subseteq \mathfrak{q}_{\mathfrak{q}} \cap R_{\mathfrak{p}}
$$

Thus, since $\mathfrak{p}_{\mathfrak{p}}$ is maximal in $R_{\mathfrak{p}}$, we have $\mathfrak{p}_{\mathfrak{p}}=\mathfrak{q}_{\mathfrak{q}} \cap R_{\mathfrak{p}}$. Similarly $\mathfrak{p}_{\mathfrak{p}}=\mathfrak{q}_{\mathfrak{q}}^{\prime} \cap R_{\mathfrak{p}}$. Whence

$$
\mathfrak{q}_{\mathfrak{q}} \cap R_{\mathfrak{p}}=\mathfrak{p}_{\mathfrak{p}}=\mathfrak{q}_{\mathfrak{q}}^{\prime} \cap R_{\mathfrak{p}}
$$

\footnotetext{
${ }^{\mathrm{b}}$ Recall that if $\mathfrak{n}, \mathfrak{n}^{\prime} \in \operatorname{Max} A$ and $\mathfrak{n} \cap Z=\mathfrak{n}^{\prime} \cap Z$ is in the Azumaya locus of $A$, then $\mathfrak{n}=\mathfrak{n}^{\prime}$.
} 
In particular, since $\mathfrak{q} \in \tilde{U}_{S / R}$,

$$
U_{S_{\mathfrak{q}} / R_{\mathfrak{p}}}:=\left\{\mathfrak{n} \in \operatorname{Max} S_{\mathfrak{q}} \mid\left(R_{\mathfrak{p}}\right)_{\mathfrak{n} \cap R_{\mathfrak{p}}}=\left(S_{\mathfrak{q}}\right)_{\mathfrak{n}}\right\}=\left\{\mathfrak{q}_{\mathfrak{q}}\right\} .
$$

Furthermore, since $\mathfrak{q} \in \tilde{U}_{S / R}$ and $\mathfrak{q}^{\prime} \in \iota^{-1} \iota(\mathfrak{p})$,

$$
S_{\mathfrak{q}}=R_{\mathfrak{p}} \subseteq S_{\mathfrak{q}^{\prime}}
$$

Thus $\mathfrak{q}^{\prime} \subseteq \mathfrak{q}$. Therefore

$$
\mathfrak{q}_{\mathfrak{q}}^{\prime} \subseteq \mathfrak{q}_{\mathfrak{q}}
$$

Whence

$$
\mathcal{Z}\left(\mathfrak{q}_{\mathfrak{q}}^{\prime}\right) \cap U_{S_{\mathfrak{q}} / R_{\mathfrak{p}}}=\left\{\mathfrak{q}_{\mathfrak{q}}\right\} \neq \emptyset .
$$

Thus, by Lemma 2.3, (5), (6), and (7) imply that

$$
\mathfrak{q}_{\mathfrak{q}}^{\prime}=\mathfrak{q}_{\mathfrak{q}} \text {. }
$$

Therefore $\mathfrak{q}^{\prime}=\mathfrak{q}_{\mathfrak{q}}^{\prime} \cap S=\mathfrak{q}_{\mathfrak{q}} \cap S=\mathfrak{q}$, which is what we wanted to show.

(1).(ii) If $\mathcal{Z}(\mathfrak{q}) \cap U_{S / R} \neq \emptyset$, then $\mathfrak{q} \in \tilde{U}_{S / R}$ by Lemma 2.2 .

(2) We now claim that $\mathfrak{n} \in W_{S / R}^{c}$ if and only if there is a point $\mathfrak{n}^{\prime} \in \operatorname{Max} S$, not equal to $\mathfrak{n}$, such that $\kappa(\mathfrak{n})=\kappa\left(\mathfrak{n}^{\prime}\right)$.

First note that for any $\mathfrak{m} \in \operatorname{Max} R$ and $\mathfrak{n} \in \kappa^{-1}(\mathfrak{m})$, we have $\mathfrak{m} \subseteq \mathfrak{m} S \subseteq$ $\sqrt{\mathfrak{m} S} \subseteq \mathfrak{n}$. Thus $\mathfrak{m} \subseteq \sqrt{\mathfrak{m} S} \cap R \subseteq \mathfrak{n} \cap R=\mathfrak{m}$. Whence

$$
\sqrt{\mathfrak{m} S} \cap R=\mathfrak{m} \text {. }
$$

Now let $\mathfrak{n} \in W_{S / R}^{c}$ and set $\mathfrak{m}:=\mathfrak{n} \cap R$. Then by definition, $\mathfrak{n} \neq \sqrt{\mathfrak{m} S}$. Since $S$ is Jacobson we have

$$
\sqrt{\mathfrak{m} S}=\bigcap_{\mathfrak{m} S \subseteq \mathfrak{q} \in \operatorname{Max} S} \mathfrak{q} .
$$

Thus there exists a maximal ideal $\mathfrak{n}^{\prime} \neq \mathfrak{n}$ of $S$ such that $\sqrt{\mathfrak{m} S} \subseteq \mathfrak{n}^{\prime}$. Whence

$$
\kappa(\mathfrak{n})=\mathfrak{n} \cap R=\mathfrak{m} \stackrel{(8)}{=} \sqrt{\mathfrak{m} S} \cap R \subseteq \mathfrak{n}^{\prime} \cap R=\kappa\left(\mathfrak{n}^{\prime}\right) .
$$

Therefore $\kappa(\mathfrak{n})=\kappa\left(\mathfrak{n}^{\prime}\right)$ by Lemma 2.1 .

Conversely, suppose there are distinct points $\mathfrak{n}, \mathfrak{n}^{\prime} \in \operatorname{Max} S$ such that $\kappa(\mathfrak{n})=$ $\kappa\left(\mathfrak{n}^{\prime}\right)$. Then $\mathfrak{n} \cap R=\mathfrak{n}^{\prime} \cap R=: \mathfrak{m}$. Therefore

$$
\sqrt{\mathfrak{m} S} \subseteq \mathfrak{n} \cap \mathfrak{n}^{\prime} \subsetneq \mathfrak{n} .
$$

(3) Suppose $U_{S / R} \neq \emptyset$. By claim (1) ८ is injective on $U_{S / R}$. Furthermore, $U_{S / R}$ is an open dense subset of Max $S$ by Proposition 2.4. Therefore Max $S$ and Max $R$ are birationally equivalent.

(4) Finally, we show that $\operatorname{dim} R=\operatorname{dim} S$. Fix $\mathfrak{n} \in U_{S / R}$ and set $\mathfrak{m}:=\mathfrak{n} \cap R$. Then

$\operatorname{dim} R \leq \operatorname{trdeg}_{k} \operatorname{Frac} R \stackrel{\text { (I) }}{=} \operatorname{trdeg}_{k} \operatorname{Frac} S \stackrel{(\mathrm{II})}{=} \operatorname{dim} S \stackrel{(\mathrm{III})}{=} \operatorname{dim} S_{\mathfrak{n}} \stackrel{(\mathrm{IV})}{=} \operatorname{dim} R_{\mathfrak{m}} \leq \operatorname{dim} R$, where (I) holds by Proposition 2.4; (II) and (III) hold since $S$ is a noetherian integral domain over $k$; and (IV) holds since $\mathfrak{n}$ is in $U_{S / R}$. 
Example 2.6. Let $S=k[x, y]$ and $R=k\left[x, x y, x y^{2}, \ldots\right]=k+x S$. For any $b \in k$, the ideals $(x, y-b) S, x S \in \operatorname{Spec} S$ satisfy

$$
(x, y-b) S \cap R=x S \cap R=\left(x, x y, x y^{2}, \ldots\right) \in \operatorname{Max} R .
$$

Thus $(x, y-b) S \in W^{c}$ by Theorem 2.5.2.

Remark 2.7. In general, $U$ need not equal $W$. Indeed, consider the algebras

$$
S=k[x] \quad \text { and } \quad R=k+x^{2} S=k\left[x^{2}, x^{3}\right] \cong k[u, v] /\left(u^{3}-v^{2}\right) .
$$

Then $U=\mathbb{A}^{1} \backslash\{0\}$ and $W=\mathbb{A}^{1}$.

The following proposition generalizes the fact that if $R$ is a finitely generated $k$-algebra and $\mathfrak{m} \in \operatorname{Max} R$, then

$$
R=k+\mathfrak{m} .
$$

Conversely, if $I$ is an ideal in $S$ and $R=k+I$, then $\mathcal{Z}(I)$ is a closed point in Spec $R$. In Corollary 3.14 below we will show that $R=k+I$ is nonnoetherian whenever the dimension of the subvariety $\mathcal{Z}(I)$ in $\operatorname{Max} S$ is nonzero.

Proposition 2.8. Let $S$ be an integral domain and a noetherian $k$-algebra. Consider a subalgebra $R^{\prime}$ of $S$, an ideal $I$ of $S$, and form the algebra

$$
R=k\left[R^{\prime}, I\right] .
$$

Then $U$ (hence $W$ ) contains the open subset $\mathcal{Z}(I)^{c}$ of $\operatorname{Max} S$.

Furthermore, if $I \subset S$ is a nonmaximal radical ideal and

$$
R=k[I]=k+I,
$$

then $U=W=\mathcal{Z}(I)^{c}$.

Proof. First suppose $R=k\left[R^{\prime}, I\right]$. We claim that if $\mathfrak{q} \in \operatorname{Spec} S$ does not contain $I$, then $R_{\mathfrak{q} \cap R}=S_{\mathfrak{q}}$; in particular,

$$
\mathcal{Z}(I)^{c} \subseteq U
$$

Set $\mathfrak{p}:=\mathfrak{q} \cap R$. Then $R_{\mathfrak{p}} \subseteq S_{\mathfrak{q}}$. To show the reverse inclusion, suppose $a \in S_{\mathfrak{q}}$. Then there is some $f, g \in S$ with $g \notin \mathfrak{q}$ such that $a=\frac{f}{g}$. Since $\mathfrak{q}$ does not contain $I$, there is some $c \in I \backslash \mathfrak{q}$. Furthermore, since $c, g \in S \backslash \mathfrak{q}$ and $\mathfrak{q}$ is prime, we have

$$
c g \in S \backslash \mathfrak{q} .
$$

Since $c \in I$, we have $c g \in I \subset R$. Whence

$$
c g \in R \backslash \mathfrak{p} .
$$

But also

$$
b:=a g c=f c \in I \subset R .
$$

Thus $a=\frac{b}{c g} \in R_{\mathfrak{p}}$. Therefore $S_{\mathfrak{q}} \subseteq R_{\mathfrak{p}}$. Consequently $R_{\mathfrak{q} \cap R}=S_{\mathfrak{q}}$. 
Now suppose $R=k[I]$, where $I$ is a nonmaximal radical ideal of $S$. Let $\mathfrak{n} \in \mathcal{Z}(I)$. Then $\mathfrak{n} \supseteq I$, so

$$
\mathfrak{n} \cap R \supseteq I \cap R=I .
$$

Whence $\mathfrak{n} \cap R=I$ since $I$ is a maximal ideal of $R$. But $\sqrt{I S}=I S=I \subsetneq \mathfrak{n}$ since $I$ is a radical ideal of $S$. Thus $\mathcal{Z}(I) \subseteq W^{c}$.

Therefore

$$
\mathcal{Z}(I) \subseteq W^{c} \stackrel{(\mathrm{I})}{\subseteq} U^{c} \stackrel{(\mathrm{II})}{\subseteq} \mathcal{Z}(I),
$$

where (I) holds by Theorem 2.5.1 and (II) holds by (9).

Remark 2.9. $U$ may properly contain $\mathcal{Z}(I)^{c}$; for example, take $R^{\prime}=S$, in which case $U=\operatorname{Max} S$.

Example 2.10 (A geometric picture). (i) Let $S=k[x, y]$ and $R=k+x S$. By Proposition 2.8, we can form $\operatorname{Max} R$ from $\operatorname{Max} S=\mathbb{A}_{k}^{2}$ by declaring the line

$$
\mathcal{Z}(x)=\{x=0\} \subset \mathbb{A}_{k}^{2}
$$

to be a single (closed) point, and all other points, $U=\{x \neq 0\}$, remain unaltered. In this description, $\mathcal{Z}(x)$ appears to be a one-dimensional, hence nonlocal, point of $\operatorname{Max} R$.

(ii) Let $S=k[x, y, z]$ and $R=k\left[x, y, y z, y z^{2}, \ldots\right]=k[x, y S]$. We can form Max $R$ from $\operatorname{Max} S=\mathbb{A}_{k}^{3}$ by declaring each line

$$
\mathcal{Z}(x-c, y)=\{x=c, y=0\} \subset \mathbb{A}_{k}^{3}
$$

to be a single point, and all other points, $U=\{y \neq 0\}$, remain unaltered. Similar to the previous example, each subvariety $\mathcal{Z}(x-c, y)$ appears to be a one-dimensional, hence nonlocal, point of $\operatorname{Max} R$.

\section{Depictions and the Geometric Dimension of a Point}

Throughout $S$ is an integral domain and a finitely generated $k$-algebra.

We introduce the following definitions with the aim of constructing a geometric theory of nonnoetherian algebras, and in particular to formalize the geometric pictures in Example 2.10. Recall that if $R$ is an integral domain and finitely generated over $k$, then the dimension of a point $\mathfrak{p} \in \operatorname{Spec} R$ coincides with the Krull dimension of $R$ minus the height of $\mathfrak{p}$,

$$
\operatorname{dim} \mathfrak{p}:=\operatorname{dim} R / \mathfrak{p}=\operatorname{dim} R-\operatorname{ht}(\mathfrak{p}) .
$$

The dimension of $\mathfrak{p}$ is then zero whenever $\mathfrak{p}$ is a maximal ideal.

\section{Definition 3.1.}

- A finitely generated $k$-algebra $S$ is a depiction of a subalgebra $R \subseteq S$ if

(i) the morphism $\iota_{S}: \operatorname{Spec} S \rightarrow \operatorname{Spec} R, \mathfrak{q} \mapsto \mathfrak{q} \cap R$, is surjective, 
(ii) for each $\mathfrak{n} \in \operatorname{Max} S, R_{\mathfrak{n} \cap R}$ is noetherian iff $R_{\mathfrak{n} \cap R}=S_{\mathfrak{n}}$, and

(iii) $U_{S / R} \neq \emptyset$.

- The geometric codimension or geometric height of $\mathfrak{p} \in \operatorname{Spec} R$ is the infimum

$$
\operatorname{ght}(\mathfrak{p}):=\inf \left\{\operatorname{ht}(\mathfrak{q}) \mid \mathfrak{q} \in \iota_{S}^{-1}(\mathfrak{p}), S \text { a depiction of } R\right\} .
$$

The geometric dimension of $\mathfrak{p}$ is the difference

$$
\operatorname{gdim} \mathfrak{p}:=\operatorname{dim} R-\operatorname{ght}(\mathfrak{p}) \text {. }
$$

Remark 3.2. Note that condition (ii) is equivalent to $U_{S / R}^{*} \subseteq U_{S / R}$. Furthermore, this condition is independent of conditions (i) and (iii). Indeed, consider

$$
S=k[x, y], \quad R=k\left[x, x y, y^{2}, y^{3}\right], \quad \text { and } \quad \mathfrak{n}=(x, y) S \in \operatorname{Max} S .
$$

Then conditions (i) and (iii) hold. ${ }^{c}$ However, $R_{\mathfrak{n} \cap R}$ is noetherian whereas $R_{\mathfrak{n} \cap R} \neq S_{\mathfrak{n}}$.

Remark 3.3. Let $S$ and $S^{\prime}$ be depictions of $R$, and let $\mathfrak{p} \in \operatorname{Spec} R$. Then in general the infimums of heights of ideals in $\iota_{S}^{-1}(\mathfrak{p})$ and $\iota_{S^{\prime}}^{-1}(\mathfrak{p})$ do not coincide. For example, let

$$
S=k[x, y, z], \quad S^{\prime}=S\left[x^{-1}\right], \quad R=k+x(y, z) S, \quad \text { and } \quad \mathfrak{m}=x(y, z) S \in \operatorname{Max} R .
$$

Then

$$
\inf \left\{\operatorname{ht}(\mathfrak{q}) \mid \mathfrak{q} \in \iota_{S}^{-1}(\mathfrak{m})\right\}=\operatorname{ht}(x S)=1
$$

whereas

$$
\inf \left\{\operatorname{ht}(\mathfrak{q}) \mid \mathfrak{q} \in \iota_{S^{\prime}}^{-1}(\mathfrak{m})\right\}=\operatorname{ht}\left((y, z) S^{\prime}\right)=2 .
$$

Remark 3.4. In [8], Schwede gives a geometric description of subalgebras of noetherian algebras which is based on the gluing of schemes. In particular, the subalgebra $k\left[x, x y, x y^{2}, \ldots\right] \subset k[x, y]$ is described as the fiber product $k[x, y] \times_{k[y]} k$ (see [8, Example 3.7]).

Lemma 3.5. If $S$ and $S^{\prime}$ are depictions of $R$, then

$$
\iota_{S}\left(U_{S / R}\right)=\iota_{S^{\prime}}\left(U_{S^{\prime} / R}\right) .
$$

Proof. We claim that

$$
\iota_{S}\left(U_{S / R}^{*}\right)=U_{R / R}^{*}
$$

Indeed, suppose $\mathfrak{m} \in U_{R / R}^{*}$. Then $R_{\mathfrak{m}}$ is noetherian. Thus, since $\iota_{S}$ is surjective, $\mathfrak{m} \in \iota_{S}\left(U_{S / R}^{*}\right)$. Conversely, suppose $\mathfrak{m} \in \iota_{S}\left(U_{S / R}^{*}\right)$. Then there is some $\mathfrak{n} \in \operatorname{Max} S$ for which $\mathfrak{n} \cap R=\mathfrak{m}$ and $R_{\mathfrak{n} \cap R}$ is noetherian. Thus $\mathfrak{m} \in U_{R / R}^{*}$. Therefore (10) holds.

\footnotetext{
c Naively it appears as though condition (i) may not hold since $x y \in x S \cap R \backslash x R$, and so there is
} no $\mathfrak{q} \in \operatorname{Spec} S$ for which $\mathfrak{q} \cap R=x R$. However, $x R$ is not prime in $R$ since $x \cdot y^{3}=(x y) y^{2}$. 
Since $S$ and $S^{\prime}$ are depictions of $R$, we have $U_{S / R}=U_{S / R}^{*}$ and $U_{S^{\prime} / R}=U_{S^{\prime} / R}^{*}$. Therefore by (10),

$$
\iota_{S}\left(U_{S / R}\right)=\iota_{S}\left(U_{S / R}^{*}\right)=U_{R / R}^{*}=\iota_{S^{\prime}}\left(U_{S^{\prime} / R}^{*}\right)=\iota_{S^{\prime}}\left(U_{S^{\prime} / R}\right) .
$$

The following lemma will be useful in Sec. 4 .

Lemma 3.6. Let $R$ be a subalgebra of $S$, and suppose $k$ is uncountable. Then the morphism $\iota: \operatorname{Spec} S \rightarrow \operatorname{Spec} R$ is surjective if and only if the morphism $\kappa$ : $\operatorname{Max} S \rightarrow \operatorname{Max} R$ is surjective.

Proof. Suppose $\kappa$ is surjective, and let $\mathfrak{p} \in \operatorname{Spec} R$. Since $S$ is a finitely generated $k$-algebra, $R$ is a countably generated $k$-algebra. By assumption $k$ is uncountable, and thus $R$ is Jacobson. Therefore, since $\mathfrak{p}$ is prime,

$$
\mathfrak{p}=\bigcap_{\mathfrak{p} \subseteq \mathfrak{m} \in \operatorname{Max} R} \mathfrak{m} .
$$

Since $\kappa$ is surjective, the ideal

$$
\mathfrak{q}:=\bigcap_{\substack{\mathfrak{n} \in \iota^{-1}(\mathfrak{m}) \text { s.t. } \\ \mathfrak{p} \subseteq \mathfrak{m} \in \operatorname{Max} R}} \mathfrak{n}
$$

satisfies $\mathfrak{q} \cap R=\mathfrak{p}$. Furthermore, $\mathfrak{q}$ is radical since it is the intersection of radical ideals. Thus, since $S$ is noetherian, the Lasker-Noether theorem implies

$$
\mathfrak{q}=\mathfrak{q}_{1} \cap \cdots \cap \mathfrak{q}_{\ell}
$$

where each $\mathfrak{q}_{i}$ is a minimal prime over $\mathfrak{q}$.

Set $\mathfrak{p}_{j}:=\mathfrak{q}_{j} \cap R$. Since $\mathfrak{p}=\mathfrak{q} \cap R \subseteq \mathfrak{q}_{j} \cap R=\mathfrak{p}_{j}$, we have $\mathfrak{p} \subseteq \mathfrak{p}_{j}$. Thus

$$
\mathfrak{p}=\sqrt{\mathfrak{p}^{\ell}} \subseteq \sqrt{\mathfrak{p}_{1} \cdots \mathfrak{p}_{\ell}} \subseteq \sqrt{\mathfrak{p}_{1} \cap \cdots \cap \mathfrak{p}_{\ell}}=\sqrt{\left(\mathfrak{q}_{1} \cap \cdots \cap \mathfrak{q}_{\ell}\right) \cap R}=\sqrt{\mathfrak{q} \cap R}=\mathfrak{p} .
$$

Whence

$$
\mathfrak{p}=\sqrt{\mathfrak{p}_{1} \cap \cdots \cap \mathfrak{p}_{\ell}} .
$$

Therefore, since $\mathfrak{p}$ is prime and each $\mathfrak{p}_{j}$ is prime (Lemma 2.1 ), there is some $1 \leq i \leq \ell$ such that $\mathfrak{p}=\mathfrak{p}_{i}$. Indeed, otherwise each $\mathfrak{p}_{i}$ would contain some $a_{i}$ not in $\mathfrak{p}$, but this is not possible since then $a_{1} \cdots a_{\ell} \in \mathfrak{p}$ and $\mathfrak{p}$ is prime. ${ }^{\mathrm{d}}$ Therefore

$$
\mathfrak{q}_{i} \cap R=\mathfrak{p}_{i}=\mathfrak{p} .
$$

It follows that $\iota$ is surjective.

${ }^{\mathrm{d}}$ In general, $\mathfrak{p}$ need not equal $\mathfrak{p}_{j}$. Indeed, consider $S=k[x, y], R=k+x S$, and the prime ideals

$$
\mathfrak{p}=\left(x y, x y^{2}, \ldots\right) R \in \operatorname{Spec} R, \quad \mathfrak{q}_{1}=x S \in \operatorname{Spec} S, \quad \mathfrak{q}_{2}=y S \in \operatorname{Spec} S .
$$

Then

$$
\mathfrak{p}=\left(\mathfrak{q}_{1} \mathfrak{q}_{2}\right) \cap R=x y S \cap R=\sqrt{(x S \cap R)(y S \cap R)}=\sqrt{\left(\mathfrak{q}_{1} \cap R\right)\left(\mathfrak{q}_{2} \cap R\right)}
$$

and $\mathfrak{p}_{2}:=\mathfrak{q}_{2} \cap R=\mathfrak{p}$, but $\mathfrak{p}_{1}:=\mathfrak{q}_{1} \cap R \neq \mathfrak{p}$. 
Lemma 3.7. Suppose $S$ is a depiction of $R$.

(1) If $\mathfrak{p} \in \operatorname{Spec} R$ and $\mathfrak{q} \in \iota^{-1}(\mathfrak{p})$, then $\operatorname{ht}(\mathfrak{q}) \leq \mathrm{ht}(\mathfrak{p})$.

(2) If $\mathfrak{m} \in \operatorname{Max} R$, then $\operatorname{ht}(\mathfrak{m})=\operatorname{dim} R$.

Proof. (1) Let $\mathfrak{p} \in \operatorname{Spec} R, \mathfrak{q} \in \iota^{-1}(\mathfrak{p})$, and set $U:=U_{S / R}$. If $\mathcal{Z}(\mathfrak{q}) \cap U \neq \emptyset$, then $S_{\mathfrak{q}}=R_{\mathfrak{p}}$ by Lemma 2.2. Whence

$$
\operatorname{ht}(\mathfrak{q})=\operatorname{dim} S_{\mathfrak{q}}=\operatorname{dim} R_{\mathfrak{p}}=\operatorname{ht}(\mathfrak{p}) .
$$

So suppose $\mathcal{Z}(\mathfrak{q}) \cap U=\emptyset$. Since $U \neq \emptyset, U$ is an open dense set by Proposition 2.4. Therefore there is a maximal chain of prime ideals in $S$ containing $\mathfrak{q}$,

$$
0 \subsetneq \mathfrak{q}_{1} \subsetneq \cdots \subsetneq \mathfrak{q}_{\ell-1} \subsetneq \mathfrak{q}_{\ell}=\mathfrak{q}
$$

such that $\mathcal{Z}\left(\mathfrak{q}_{i}\right) \cap U \neq \emptyset$ for each $1 \leq i \leq \ell-1$.

Set $\mathfrak{p}_{i}:=\mathfrak{q}_{i} \cap R \in \operatorname{Spec} R$. Then by Lemma $2.2, R_{\mathfrak{p}_{i}}=S_{\mathfrak{q}_{i}}$. In particular,

$$
\operatorname{ht}\left(\mathfrak{p}_{\ell-1}\right)=\operatorname{dim} R_{\mathfrak{p}_{\ell-1}}=\operatorname{dim} S_{\mathfrak{q}_{\ell-1}}=\operatorname{ht}\left(\mathfrak{q}_{\ell-1}\right) .
$$

Furthermore, by the contrapositive of Lemma 2.3,

$$
\mathfrak{p}_{\ell-1} \subsetneq \mathfrak{p} .
$$

Therefore $h t(\mathfrak{q}) \leq \mathrm{ht}(\mathfrak{p})$.

(2) Let $\mathfrak{m} \in \operatorname{Max} R$. Since $\iota$ is surjective, there is some $\mathfrak{q} \in \operatorname{Spec} S$ such that $\iota(\mathfrak{q})=\mathfrak{m}$. Let $\mathfrak{n}$ be a maximal ideal of $S$ containing $\mathfrak{q}$. Then $\mathfrak{m}=\mathfrak{q} \cap R \subseteq \mathfrak{n} \cap R$, and so $\mathfrak{m}=\mathfrak{n} \cap R$ since $\mathfrak{m}$ is maximal. Therefore

$$
\operatorname{dim} R \stackrel{(\mathrm{I})}{=} \operatorname{dim} S \stackrel{(\mathrm{II})}{=} \mathrm{ht}(\mathfrak{n}) \stackrel{(\mathrm{III})}{\leq} \mathrm{ht}(\mathfrak{m}) \leq \operatorname{dim} R .
$$

Indeed, (I) holds by Theorem 2.5.4; (II) holds since $S$ an integral domain; and (III) holds by claim (1).

Theorem 3.8. Suppose $R$ admits a depiction and let $\mathfrak{p} \in \operatorname{Spec} R$. Then

$$
\operatorname{ght}(\mathfrak{p}) \leq \operatorname{ht}(\mathfrak{p}),
$$

with equality if there is a depiction $S$ of $R$ and $\mathfrak{q} \in \iota_{S}^{-1}(\mathfrak{p})$ such that $\mathcal{Z}(\mathfrak{q}) \cap U_{S / R} \neq \emptyset$. Furthermore,

(1) If $R$ is noetherian, then

$$
\operatorname{ght}(\mathfrak{p})=\operatorname{ht}(\mathfrak{p})
$$

(2) If $\mathfrak{m} \in \operatorname{Max} R, \mathfrak{q} \in \iota_{S}^{-1}(\mathfrak{m})$, and $\operatorname{dim} \mathcal{Z}(\mathfrak{q}) \geq 1$, then

$$
\operatorname{ght}(\mathfrak{m}) \neq \operatorname{ht}(\mathfrak{m}) .
$$


Proof. The inequality (11) holds by Lemma 3.7.1. Furthermore, if $\mathcal{Z}(\mathfrak{q}) \cap U_{S / R} \neq \emptyset$, then $\operatorname{ght}(\mathfrak{p})=\operatorname{ht}(\mathfrak{p})$ by Lemma 3.5.

Suppose $R$ is noetherian. Then $R$ is a depiction of itself with $U_{R / R}=\operatorname{Max} R$. Therefore claim (1) holds as a particular case of the previous paragraph.

Now assume the hypotheses of claim (2). Then

$$
\operatorname{ght}(\mathfrak{m}) \stackrel{(\mathrm{I})}{\leq} \operatorname{ht}(\mathfrak{q}) \stackrel{(\mathrm{II})}{<} \operatorname{dim} S \stackrel{(\mathrm{III})}{=} \operatorname{dim} R \stackrel{(\mathrm{IV})}{=} \operatorname{ht}(\mathfrak{m}) .
$$

Indeed, (I) holds by Definition 3.1; (II) holds since $\operatorname{dim} \mathcal{Z}(\mathfrak{q}) \geq 1$; (III) holds by Theorem 2.5.4; and (IV) holds by Lemma 3.7.2.

A depiction, by definition, is a finitely generated $k$-algebra. However, to capture a similar notion for local rings we introduce the following.

Definition 3.9. Suppose $S$ is a depiction of $R$ and let $\mathfrak{q} \in \operatorname{Spec} S$. Then we say $S_{\mathfrak{q}}$ is a local depiction of $R_{\mathfrak{q} \cap R}$.

Remark 3.10. A local depiction may not satisfy conditions (i) or (iii) in Definition 3.1. For example, consider $S=k[x, y]$ and $R=k+x S$. Then the local ring $S_{x S}$ is a noetherian overring of the local ring $R_{x S}$, but $U_{S_{x S} / R_{x S}}=\emptyset$.

Furthermore, although $\iota_{S}: \operatorname{Spec} S \rightarrow \operatorname{Spec} R$ is surjective, the morphism $\iota_{S_{x S}}$ : Spec $S_{x S} \rightarrow$ Spec $R_{x S}$ is not surjective. Indeed, there are only two prime ideals of $S$ contained in $x S$,

$$
0 \subsetneq x S
$$

However, there are three prime ideals of $R$ contained in $x S$, namely

$$
0 \subsetneq y S \cap R \subsetneq x S \cap R=x S .
$$

(These ideals are prime by Lemma 2.1.) Therefore

$$
\iota_{S_{x S}}: \operatorname{Spec} S_{x S} \rightarrow \operatorname{Spec} R_{x S}
$$

is not surjective.

Example 3.11. We give an explicit example where the height and geometric codimension of an ideal do not coincide. Let $S=k[x, y]$ and $R=k+x S$. Then the chains (12) and (13) imply

$$
\operatorname{ght}(x S \cap R)=1 \neq 2=\operatorname{ht}(x S \cap R) .
$$

The following two theorems establish relationships between depictions, geometric dimension, and noetherianity.

Theorem 3.12. Suppose $S$ is a depiction of $R$. Then the following conditions are equivalent:

(1) $R$ is noetherian. 
(2) $U_{S / R}=\operatorname{Max} S$.

(3) $R=S$.

In particular, if $R$ is noetherian, then its only depiction is itself.

Proof. $(2 \Rightarrow 1,3)$ Suppose $U_{S / R}=\operatorname{Max} S$. Then

$$
R \stackrel{(\mathrm{I})}{=} \bigcap_{\mathfrak{m} \in \operatorname{Max} R} R_{\mathfrak{m}} \stackrel{(\mathrm{II})}{=} \bigcap_{\mathfrak{n} \in \operatorname{Max} S} R_{\mathfrak{n} \cap R} \stackrel{(\mathrm{III})}{=} \bigcap_{\mathfrak{n} \in \operatorname{Max} S} S_{\mathfrak{n}} \stackrel{(\mathrm{IV})}{=} S .
$$

Indeed, (I) and (IV) hold since $R$ and $S$ are unital commutative rings; (II) holds since $S$ is a depiction of $R$, whence $\kappa: \operatorname{Max} S \rightarrow \operatorname{Max} R$ is surjective; and (III) holds since $U_{S / R}=\operatorname{Max} S$. Therefore $R=S$ is noetherian.

$(2 \Rightarrow 3)$ Alternatively, suppose there is some $g \in S \backslash R$. Then the fractional ideal $(R: g)_{R}$ is proper, and is thus contained in some maximal ideal $\mathfrak{m} \in \operatorname{Max} R$. Let $\mathfrak{n} \in \kappa^{-1}(\mathfrak{m})$. Then $g \in S_{\mathfrak{n}} \backslash R_{\mathfrak{m}}$. Thus $\mathfrak{n} \in U_{S / R}^{c}$, and therefore $U_{S / R}^{c} \neq \emptyset$.

$(1 \Rightarrow 2)$ Suppose $R$ is noetherian. Then $R$ is a depiction of itself. Let $S$ be another depiction of $R$. Then

$$
\iota_{S}\left(U_{S / R}\right) \subseteq \iota_{S}(\operatorname{Max} S) \subseteq \operatorname{Max} R=\iota_{R}\left(U_{R / R}\right) \stackrel{(\mathrm{I})}{=} \iota_{S}\left(U_{S / R}\right),
$$

where (I) holds by Lemma 3.5. Whence $\iota_{S}\left(U_{S / R}\right)=\iota_{S}(\operatorname{Max} S)$. But $\iota_{S}$ is injective on $U_{S / R}$ by Theorem 2.5.1. Therefore $U_{S / R}=\operatorname{Max} S$.

$(3 \Rightarrow 1)$ If $R=S$, then $R$ is trivially noetherian.

Theorem 3.13. If $\operatorname{Max} R$ contains a closed point of positive geometric dimension, then $R$ is nonnoetherian. Conversely, if $R$ is nonnoetherian, depicted by $S$, and there is some $\mathfrak{m} \in \iota\left(U_{S / R}^{c}\right)$ such that $\sqrt{\mathfrak{m} S}=\mathfrak{m}$, then $\operatorname{Max} R$ contains a closed point of positive geometric dimension.

Proof. (i) Suppose $R$ contains a closed point of positive geometric dimension. Then $R$ admits a depiction $S$. Let $I$ be a radical ideal of $S$ such that $I \cap R=: \mathfrak{m}$ is a maximal ideal of $R$ and $\operatorname{dim} \mathcal{Z}(I) \geq 1$. Let $\mathfrak{q} \in \operatorname{Spec} S$ be a minimal prime over $I$. Then $\operatorname{dim} \mathcal{Z}(\mathfrak{q}) \geq 1$. Furthermore, $\mathfrak{m}=I \cap R \subseteq \mathfrak{q} \cap R \neq R$ implies $\mathfrak{m}=\mathfrak{q} \cap R$ since $\mathfrak{m}$ is maximal. Thus by Theorem 3.8.2,

$$
\operatorname{ght}(\mathfrak{m}) \neq \operatorname{ht}(\mathfrak{m}) .
$$

Therefore $R$ is not noetherian by Theorem 3.8.1.

(ii) Suppose the hypotheses hold, and $\operatorname{dim} U_{S / R}^{c}=0$. We claim that $R$ is noetherian. More specifically, we claim that $R$ is a finitely generated $k$-algebra. To show this, it suffices to show that $S$ is a finitely generated $R$-module by the Artin-Tate lemma.

By the Lasker-Noether theorem, there are ideals $\mathfrak{n}_{1}, \ldots, \mathfrak{n}_{\ell} \in \operatorname{Spec} S$ such that

$$
\mathfrak{m}=\sqrt{\mathfrak{m} S}=\mathfrak{n}_{1} \cap \cdots \cap \mathfrak{n}_{\ell}
$$


Whence

$$
\mathfrak{m}=\mathfrak{m} \cap R=\left(\mathfrak{n}_{1} \cap \cdots \cap \mathfrak{n}_{\ell}\right) \cap R=\left(\mathfrak{n}_{1} \cap R\right) \cap \cdots \cap\left(\mathfrak{n}_{\ell} \cap R\right) .
$$

Since $\mathfrak{m}$ is maximal in $R$ and each $\mathfrak{n}_{i} \cap R$ is proper, we have

$$
\mathfrak{n}_{1} \cap R=\cdots=\mathfrak{n}_{\ell} \cap R=\mathfrak{m} .
$$

Therefore, since $\operatorname{dim} U_{S / R}^{c}=0$, each $\mathfrak{n}_{i}$ is a maximal ideal of $S$.

Let $x_{1}, \ldots, x_{t}$ be a minimal generating set for $S$ over $k$. Since $k$ is algebraically closed, for each $1 \leq i \leq \ell$ and $1 \leq j \leq t$ there are scalars $\alpha_{i j} \in k$ such that

$$
x_{j}-\alpha_{i j} \in \mathfrak{n}_{i} .
$$

Set the degree of each $x_{j}$ to be 1 . Denote by $\mathcal{M}$ the set of monomials in the variables $x_{1}, \ldots, x_{t}$, with coefficient 1 and degree at most $\ell-1$. Since $S$ is a finitely generated $k$-algebra, $\mathcal{M}$ is finite.

We proceed by induction to show that as $k$-spaces,

$$
S=R+\sum_{m \in \mathcal{M}} k m .
$$

Base case. Consider a monomial $x_{j_{1}} \cdots x_{j_{\ell}}$ of degree $\ell$. Set

$$
r:=\left(x_{j_{1}}-\alpha_{1 j_{1}}\right)\left(x_{j_{2}}-\alpha_{2 j_{2}}\right) \cdots\left(x_{j_{\ell}}-\alpha_{\ell j_{\ell}}\right) \quad \text { and } \quad h:=x_{j_{1}} \cdots x_{j_{\ell}}-r .
$$

Then $r$ is in $\mathfrak{n}_{1} \cap \cdots \cap \mathfrak{n}_{\ell}=\mathfrak{m} \subset R$ by (14). Furthermore, $h$ has degree at most $\ell-1$. Thus $h$ is in $\sum_{m \in \mathcal{M}} k m$ by the definition of $\mathcal{M}$. Therefore $x_{j_{1}} \cdots x_{j_{\ell}}=r+h$ is in $R+\sum_{m \in \mathcal{M}} k m$.

Inductive step. Now suppose all monomials of degree at most $d-1$ are in $R+$ $\sum_{m \in \mathcal{M}} k m$, and consider a monomial $x_{j_{1}} \cdots x_{j_{d}}$ of degree $d$. Set

$$
r:=\left(x_{j_{1}}-\alpha_{1 j_{1}}\right) \cdots\left(x_{j_{\ell}}-\alpha_{\ell_{\ell}}\right) x_{j_{\ell+1}} \cdots x_{j_{d}} \quad \text { and } \quad h:=x_{j_{1}} \cdots x_{j_{d}}-r .
$$

Then $r$ is in $\mathfrak{n}_{1} \cap \cdots \cap \mathfrak{n}_{\ell}=\mathfrak{m} \subset R$, again by (14). Furthermore, $h$ has degree at most $d-1$. Thus $h$ is in $R+\sum_{m \in \mathcal{M}} k m$ by the induction hypothesis. Therefore $x_{j_{1}} \cdots x_{j_{d}}=r+h$ is in $R+\sum_{m \in \mathcal{M}} k m$. This proves our claim (15).

But

$$
R+\sum_{m \in \mathcal{M}} k m \subseteq R+\sum_{m \in \mathcal{M}} R m .
$$

Thus as $R$-modules,

$$
S=R+\sum_{m \in \mathcal{M}} R m
$$

Therefore $S$ is a finitely generated $R$-module since $\mathcal{M}$ is finite.

The following corollary is immediate.

Corollary 3.14. Let I be a radical ideal of a finitely generated $k$-algebra $S$. Then the ring $R=k+I$ is nonnoetherian if and only if $\operatorname{dim} \mathcal{Z}(I) \geq 1$. 
Example 3.15. Let $S$ be a finitely generated $k$-algebra, and let $\mathfrak{n}_{1}, \mathfrak{n}_{2}, \ldots, \mathfrak{n}_{\ell}$ be a finite set of maximal ideals of $S$. Then by Corollary 3.14, the ring

$$
R=k+\sqrt{\mathfrak{n}_{1} \mathfrak{n}_{2} \cdots \mathfrak{n}_{\ell}}
$$

is noetherian. Furthermore, the $\ell$ points $\mathfrak{n}_{1}, \ldots, \mathfrak{n}_{\ell}$ of Max $S$ are identified as one point in $\operatorname{Max} R$, and all other points are unaltered by Proposition 2.8,

$$
U_{S / R}=\mathcal{Z}\left(\mathfrak{n}_{1} \cdots \mathfrak{n}_{\ell}\right)^{c} .
$$

In particular, the variety $\operatorname{Max} R$ is nonlocal. $R$ is therefore an example of a noetherian ring with nonlocal geometry, although its one nonlocal point is zero dimensional.

The following proposition characterizes the assumptions in the converse implication of Theorem 3.13 .

Proposition 3.16. If there is a point $\mathfrak{m} \in \iota\left(U_{S / R}^{c}\right)$ satisfying $\mathfrak{m} S=\mathfrak{m}$, then

$$
U_{S / R}^{c}=\{\mathfrak{m}\} .
$$

If additionally $S$ is a depiction of $R$, then $R$ is an isolated nonnoetherian singularity.

Proof. Suppose $\mathfrak{m}=\mathfrak{m} S$. Consider $\mathfrak{m}^{\prime} \in \operatorname{Max} R \backslash\{\mathfrak{m}\}$. Fix $\mathfrak{n}^{\prime} \in \iota^{-1}\left(\mathfrak{m}^{\prime}\right)$ and $g \in$ $\mathfrak{m} \backslash \mathfrak{m}^{\prime}$. Since $\mathfrak{m}=\mathfrak{m} S$, the ideal $S g$ is in $\mathfrak{m} \subset R$. Thus

$$
S=S g \cdot g^{-1} \subset R_{\mathfrak{m}^{\prime}}
$$

Whence $R_{\mathfrak{m}^{\prime}}=S_{\mathfrak{n}^{\prime}}$. Thus $\mathfrak{m}^{\prime} \in \iota\left(U_{S / R}\right)$. Therefore (16) holds.

If $S$ is a depiction of $R$, then $U_{S / R}^{*}=U_{S / R}$, and so (16) implies that $R$ is an isolated nonnoetherian singularity.

Question 3.17. Is there a nonnoetherian algebra (which admits a depiction) such that all of its closed points have geometric dimension zero? By Proposition 3.16, such an algebra would necessarily be a nonisolated nonnoetherian singularity. Or is it the case that $R$ is nonnoetherian if and only if $\operatorname{Max} R$ contains a closed point of positive geometric dimension?

Example 3.18. Consider the algebras

$$
S=k[x, y], \quad R=k+x S, \quad R^{\prime}=k[x, x y] .
$$

Set $\mathfrak{n}:=(x, y) S$. By Example 3.11, the closed point $\mathfrak{n} \cap R$ of Max $R$ has geometric dimension 1. Naively it appears that the closed point $\mathfrak{n} \cap R^{\prime}$ of Max $R^{\prime}$ should also have geometric dimension 1, contrary to the claim of Theorem 3.13 since $R^{\prime}$ is noetherian. However, although $R$ is depicted by $S, R^{\prime}$ is not. Indeed, the morphism $\iota: \operatorname{Spec} S \rightarrow \operatorname{Spec} R^{\prime}$ is not surjective: the ideal $x R^{\prime}$ is prime in $R^{\prime}$, but

$$
\iota^{-1}\left(x R^{\prime}\right)=\emptyset \text {. }
$$

In contrast, the ideal $x R$ is not prime in $R$ since $x \cdot x y^{2}=x y \cdot x y$.

In view of Lemma 3.6, consider the maximal ideals

$$
\mathfrak{m}_{\alpha}:=(x, x y-\alpha) R^{\prime} \in \operatorname{Max} R^{\prime}
$$


with $\alpha \in k$. Then

$$
x R^{\prime}=\bigcap_{\alpha \in k} \mathfrak{m}_{\alpha}
$$

Furthermore, if $\alpha \neq 0$, then $1 \in \mathfrak{m}_{\alpha} S$. Thus if $\mathfrak{n} \in \operatorname{Max} S$ satisfies $\mathfrak{n} \cap R=\mathfrak{m}_{\alpha}$, then

$$
1 \in \mathfrak{m}_{\alpha} S \subseteq \mathfrak{n},
$$

which is not possible. Therefore the morphism $\kappa: \operatorname{Max} S \rightarrow \operatorname{Max} R^{\prime}$ is also not surjective.

We say a depiction $S$ of $R$ is maximal (resp. minimal) if $S$ is not contained in (resp. does not contain) any other depiction of $R$.

Proposition 3.19. (1) Minimal depictions do not exist in general.

(2) Maximal depictions are not unique in general.

Proof. (1) We first show that minimal depictions need not exist. Let $S=k[x, y]$ and $R=k+x S$. For $\ell \in \mathbb{N}$, set

$$
S_{\ell}:=R\left[y^{\ell}, y^{\ell+1}, y^{\ell+2}, \ldots\right]=k\left[x, x y, x y^{2}, \ldots, x y^{\ell-1}, y^{\ell}, y^{\ell+1}, \ldots, y^{2 \ell-1}\right] .
$$

Then each $S_{\ell}$ is a depiction of $R$. However,

$$
S_{\ell+1} \subsetneq S_{\ell} \quad \text { and } \quad R=\bigcap_{\ell \geq 1} S_{\ell} .
$$

(2) We now show that maximal depictions need not be unique. Let $T=k[x, y, z]$ and $R=k+x y T$. We claim that the overrings

$$
S:=T\left[x^{-1}\right] \quad \text { and } \quad S^{\prime}:=T\left[y^{-1}\right]
$$

are both depictions of $R$.

Indeed, $U_{S / R}$ is nonempty: Let $a, b \in k^{*}$. Then $x^{-1}$ and each $f \in T$ are in $R_{(x-a, y-b, z) S \cap R}$ since

$$
x^{-1}=x y^{2} \cdot \frac{1}{(x y)^{2}} \quad \text { and } \quad f=x y f \cdot \frac{1}{x y} .
$$

Thus

$$
R_{(x-a, y-b, z) S \cap R}=S_{(x-a, y-b, z) S} .
$$

Therefore the maximal ideal $(x-a, y-b, z) S$ is in $U_{S / R}$. Similarly $U_{S^{\prime} / R}$ is nonempty.

It is straightforward to check that $\iota_{S}: \operatorname{Spec} S \rightarrow \operatorname{Spec} R$ and $\iota_{S^{\prime}}: \operatorname{Spec} S^{\prime} \rightarrow$ Spec $R$ are both surjective by Lemma 3.6 and noting that

$$
y S \cap R=x S^{\prime} \cap R=x y T \in \operatorname{Max} R .
$$

Finally, the minimal proper overring $T\left[x^{-1}, y^{-1}\right]$ of $S$ and $S^{\prime}$ is not a depiction of $R: T\left[x^{-1}, y^{-1}\right]$ has no maximal ideal $\mathfrak{n}$ satisfying $\mathfrak{n} \cap R=x y T$. Therefore

$$
\iota_{T\left[x^{-1}, y^{-1}\right]}: \operatorname{Spec} T\left[x^{-1}, y^{-1}\right] \rightarrow \operatorname{Spec} R
$$

is not surjective. 


\section{Noncommutative Nonnoetherian Geometry}

Throughout $k$ is an uncountable algebraically closed field of characteristic zero; $A=k Q / I$ is a quiver algebra with finite quiver $Q$ and $I \subset k Q_{\geq 1}$; and $B$ is a finitely generated integral domain over $k$. We say an element $c \in A=k Q / I$ is a cycle (resp. path) if there is a cycle (resp. path) $c^{\prime} \in k Q$ such that $c=c^{\prime}+I$.

Denote by $E_{j i} \in M_{\left|Q_{0}\right|}(B)$ the matrix with a 1 in the $(j i)$ th slot and zeros elsewhere. Let $\tau: A \rightarrow M_{\left|Q_{0}\right|}(B)$ be an algebra homomorphism such that $\tau\left(e_{i}\right)=$ $E_{i i}$ for each $i \in Q_{0}$. For $p \in e_{j} A e_{i}$, denote by $\bar{\tau}(p) \in B$ the single nonzero entry of the matrix $\tau(p)$. For each $i, j \in Q_{0}, \bar{\tau}$ defines a $k$-linear map $\bar{\tau}: e_{j} A e_{i} \rightarrow B$. Set

$$
\begin{gathered}
R:=k\left[\cap_{i \in Q_{0}} \bar{\tau}\left(e_{i} A e_{i}\right)\right] \subseteq B, \\
S:=k\left[\cup_{i \in Q_{0}} \bar{\tau}\left(e_{i} A e_{i}\right)\right] \subseteq B .
\end{gathered}
$$

Recall the definition of an impression given in Definition 1.1. An impression is useful in part because it determines the center $Z$ of $A$ [1, Lemma 2.1]:

$$
Z \cong\left\{f \in B \mid f 1_{d} \in \operatorname{im} \tau\right\} \subseteq B
$$

Theorem 4.1. Suppose $\tau: A \hookrightarrow M_{\left|Q_{0}\right|}(B)$ is an impression of $A$ with $B$ an integral domain and $\tau\left(e_{i}\right)=E_{i i}$ for each $i \in Q_{0}$. Then $U_{S / R} \neq \emptyset$. Furthermore, if $U_{S / R}^{*} \subseteq U_{S / R}$, then

(1) The center $Z$ of $A$ is isomorphic to $R$ and is depicted by $S$.

(2) The statements

(a) $R=S$;

(b) $A$ is a finitely generated $Z$-module;

(c) $Z$ is noetherian;

(d) A is noetherian;

satisfy the equivalences

$$
(\mathrm{a}) \Longleftrightarrow(\mathrm{b}) \Longleftrightarrow(\mathrm{c}) \rightleftharpoons(\mathrm{d})
$$

where $(\star)$ holds if the $\bar{\tau}$-image of a path is a monomial in $B$ and $I$ is generated by binomials in the paths of $Q$.

In Example 4.5 below we show that the assumption $U_{S / R}^{*} \subseteq U_{S / R}$ is independent from the impression assumption.

Proof. (1.i) We first show that the center of $A$ is isomorphic to $R$. Set $d:=\left|Q_{0}\right|$. Clearly

$$
\left\{f \in B \mid f 1_{d} \in \operatorname{im} \tau\right\} \subseteq R
$$


So suppose $f \in R$. Then for each $i \in Q_{0}$ there is some $c_{i} \in e_{i} A e_{i}$ such that $\bar{\tau}\left(c_{i}\right)=f$. Thus $\tau\left(\sum_{i \in Q_{0}} c_{i}\right)=f 1_{d}$. Whence $\left\{f \in B \mid f 1_{d} \in \operatorname{im} \tau\right\} \supseteq R$. Thus by $(17)$,

$$
Z \cong\left\{f \in B \mid f 1_{d} \in \operatorname{im} \tau\right\}=R .
$$

(1.ii) We now show that $R$ is depicted by $S$.

(1.ii.I) $S$ is a finitely generated domain over $k$. By assumption $Q$ is finite. Thus there are a finite number of nonvertex cycles in $Q$ without cyclic proper subpaths (not viewed modulo $I$ ), say $c_{1}, \ldots, c_{\ell}$. Note that each cycle has length at most $\left|Q_{0}\right|$.

We claim that

$$
S=k\left[\bar{\tau}\left(c_{1}\right), \ldots, \bar{\tau}\left(c_{\ell}\right)\right] .
$$

It suffices to show that the $\bar{\tau}$-image of each nonvertex cycle with a cyclic proper subpath is contained in $k\left[\bar{\tau}\left(c_{1}\right), \ldots, \bar{\tau}\left(c_{\ell}\right)\right]$. So suppose $d$ is such a cycle. Then $d$ has a cyclic proper subpath $c$ with no cyclic proper subpaths (again, not viewed modulo $I)$. Thus there are paths $d_{1}, d_{2}$ such that $d=d_{2} c d_{1}$. Since $c$ is a cycle, $d_{2} d_{1}$ is also a cycle. Therefore, since $\tau$ is an algebra homomorphism and $B$ is commutative,

$$
\bar{\tau}(d)=\bar{\tau}\left(d_{2} c d_{1}\right)=\bar{\tau}\left(d_{2}\right) \bar{\tau}(c) \bar{\tau}\left(d_{1}\right)=\bar{\tau}(c) \bar{\tau}\left(d_{2}\right) \bar{\tau}\left(d_{1}\right)=\bar{\tau}(c) \bar{\tau}\left(d_{2} d_{1}\right) .
$$

The length of the cycle $d_{2} d_{1}$ is strictly less than the length of $d$ since $c$ is a nonvertex cycle. Our claim (18) then follows by induction on the length of the cycles.

Furthermore, $S$ is a domain since it is a subalgebra of the domain $B$.

(1.ii.II) The set $U_{S / R}$ is nonempty. By [1, Lemma 2.4], the dimension vector for the simple $A$-modules of maximal $k$-dimension is $1^{Q_{0}}$. Thus there exists a path $p_{j i} \notin I$ between any two vertices $i, j$ of $Q$. The cycle $b:=p_{1 d} \cdots p_{32} p_{21}$ then contains each vertex as a subpath. Furthermore, since $\tau$ is injective, $\bar{\tau}\left(p_{j i}\right) \neq 0$. Thus, since $B$ is an integral domain and $\tau$ is an algebra homomorphism,

$$
\bar{\tau}(b)=\bar{\tau}\left(p_{1 d} \cdots p_{32} p_{21}\right)=\bar{\tau}\left(p_{1 d}\right) \cdots \bar{\tau}\left(p_{32}\right) \bar{\tau}\left(p_{21}\right) \neq 0 .
$$

Whence $\bar{\tau}(b) \neq 0$.

Fix $i \in Q_{0}$, and let $c_{i} \in e_{i} A e_{i}$ be an arbitrary cycle. For each $j \in Q_{0}$, denote by $b_{j}$ and $d_{j}$ the respective cycles obtained by cyclically permuting $b$ and $d_{i}:=b c_{i}$ so that their heads and tails are at $j$. Then $\bar{\tau}\left(b_{j}\right)=\bar{\tau}(b)=: \beta$ and $\bar{\tau}\left(d_{j}\right)=\bar{\tau}\left(d_{i}\right)=$ $\bar{\tau}\left(c_{i}\right) \beta$, since $\tau$ is an algebra homomorphism on $e_{i} A e_{i}$. Therefore $\beta$ and $\bar{\tau}\left(c_{i}\right) \beta$ are in $R$.

Fix $\mathfrak{b} \in \mathcal{Z}(\beta)^{c} \subset \operatorname{Max} B$. By Lemma $2.1, \mathfrak{n}:=\mathfrak{b} \cap S$ and $\mathfrak{m}:=\mathfrak{n} \cap R$ are maximal ideals of $S$ and $R$ respectively. Furthermore, $\beta \in R$ is invertible in the localization $R_{\mathfrak{m}}$. Consequently

$$
\bar{\tau}\left(c_{i}\right)=\bar{\tau}\left(c_{i}\right) \beta \cdot \beta^{-1} \in R_{\mathfrak{m}} .
$$

Since $c_{i}$ was arbitrary, $S_{\mathfrak{n}} \subseteq R_{\mathfrak{m}}$. Whence $S_{\mathfrak{n}}=R_{\mathfrak{m}}$.

(1.ii.III) The map $\iota: \operatorname{Spec} S \rightarrow \operatorname{Spec} R, \mathfrak{q} \mapsto \mathfrak{q} \cap R$, is surjective. By claim (1.ii.a), $S$ is a finitely generated $k$-algebra. Thus by Lemma 3.6, it suffices to show 
$\kappa: \operatorname{Max} S \rightarrow \operatorname{Max} R, \mathfrak{n} \mapsto \mathfrak{n} \cap R$, is surjective. Let $\mathfrak{m} \in \operatorname{Max} R$. By the definition of impression, the morphism $\operatorname{Max} B \rightarrow \operatorname{Max} \tau(Z), \mathfrak{b} \mapsto \mathfrak{b} 1_{d} \cap \tau(Z)$, is surjective. Thus there is some $\mathfrak{b} \in \operatorname{Max} B$ such that $\mathfrak{b} \cap R=\mathfrak{m}$. By Lemma 2.1, $\mathfrak{n}:=\mathfrak{b} \cap S$ is in $\operatorname{Max} S$. Furthermore, $\mathfrak{n}$ satisfies

$$
\mathfrak{n} \cap R=(\mathfrak{b} \cap S) \cap R=\mathfrak{m} .
$$

Thus $\kappa$ is surjective.

(1.ii.IV) Claims (1.ii.I, II, III), together with the assumption $U_{S / R}^{*} \subseteq U_{S / R}$, imply that $S$ is a depiction of $R$.

(2. a $\Leftrightarrow$ c) Follows from claim (1) and Theorem 3.12.2.

(2. $\mathrm{a} \Rightarrow \mathrm{b}, \mathrm{d})$ Follows from [1, Theorem 2.11].

$(2 \mathrm{~b} \Rightarrow \mathrm{a})$ Suppose $R \neq S$. Then $R$ is an infinitely generated $k$-algebra by Theorem 3.12.1. Furthermore, $S$ is a finitely generated $k$-algebra by claim (1.ii.I). Thus $S$ is an infinitely generated $R$-module by the Artin-Tate lemma. But $\tau$ is injective, $\left|Q_{0}\right|<\infty$, and $Z \cong R$ by claim (1). Therefore $\bigoplus_{i \in Q_{0}} e_{i} A e_{i}$ is an infinitely generated $Z$-module. Whence $A$ is an infinitely generated $Z$-module.

$(2$. $\mathrm{d} \Rightarrow \mathrm{a})$ Suppose $R \neq S$, and the conditions $(\star)$ hold. As was shown in (b $\Rightarrow$ a), $S$ is an infinitely generated $R$-module. Thus there is a cycle $q$ and vertex $i$ such that for each $n \geq 1$,

$$
\bar{\tau}(q)^{n} \in S \backslash \bar{\tau}\left(e_{i} A e_{i}\right) .
$$

Since $\tau$ is an impression of $A$, there is a path $p_{1}$ in $e_{\mathrm{t}(q)} A e_{i}$ and a path $p_{2}$ in $e_{i} A e_{\mathrm{t}(q)}$. Assume to the contrary that the chain of ideals

$$
0 \subsetneq\left(p_{2} p_{1}\right) \subsetneq\left(p_{2} p_{1}, p_{2} q p_{1}\right) \subsetneq\left(p_{2} p_{1}, p_{2} q p_{1}, p_{2} q^{2} p_{1}\right) \subsetneq \cdots
$$

in $e_{i} A e_{i}$ terminates. Then, since $I$ is generated by binomials in the paths of $Q$, there is some $n>m \geq 0$ and a cycle $a \in e_{i} A e_{i}$ such that

$$
p_{2} q^{n} p_{1}=a p_{2} q^{m} p_{1}
$$

Whence

$$
\bar{\tau}(q)^{n} \bar{\tau}\left(p_{2} p_{1}\right)=\bar{\tau}\left(p_{2} q^{n} p_{1}\right)=\bar{\tau}\left(a p_{2} q^{m} p_{1}\right)=\bar{\tau}(a) \bar{\tau}(q)^{m} \bar{\tau}\left(p_{2} p_{1}\right) .
$$

Thus since $B$ is an integral domain,

$$
\bar{\tau}(q)^{n-m}=\bar{\tau}(a) \in \bar{\tau}\left(e_{i} A e_{i}\right) .
$$

But this contradicts our choice of $q$.

Thus the vertex corner ring $e_{i} A e_{i}$ is nonnoetherian. Therefore $A$ is nonnoetherian.

Remark 4.2. The role of $S$ is new: $S$ is a commutative ring obtained from $A$ that in most cases is not a central subring of $A$, but is closely related to the geometry of the center $Z$ of $A$. By Theorem 4.1.2, if $Z$ is noetherian, then $S$ is isomorphic to $Z$, and if $Z$ is nonnoetherian, then $S$ properly contains $Z$. 


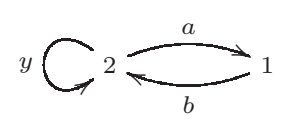

Fig. 1.

Example 4.3. Consider the quiver algebra

$$
A=k Q /\langle y b a-b a y\rangle
$$

with quiver given in Fig. 1. $A$ admits the impression $(\tau, B=k[x, y])$ where $\tau\left(e_{i}\right)=$ $E_{i i}$ for $i=1,2$, and

$$
\bar{\tau}(a)=1, \quad \bar{\tau}(b)=x, \quad \bar{\tau}(y)=y .
$$

Recall our motivating example in Sec. $2, S^{\prime}=k[x, y]$ and $R^{\prime}=k+x S^{\prime}$. By (17), the center of $A$ is isomorphic to

$$
R=k\left[\bar{\tau}\left(e_{1} A e_{1}\right) \cap \bar{\tau}\left(e_{2} A e_{2}\right)\right]=k+x S^{\prime}=R^{\prime}
$$

and is depicted by

$$
S=k\left[\bar{\tau}\left(e_{1} A e_{1}\right) \cup \bar{\tau}\left(e_{2} A e_{2}\right)\right]=k[x, y]=S^{\prime} .
$$

By Theorem 4.1.2, $A$ and $Z$ are nonnoetherian, and $A$ is an infinitely generated $Z$-module.

We recall a homological characterization of smoothness, in the both the commutative and noncommutative settings. Let $R$ be a noetherian integral domain and let $\mathfrak{p} \in \operatorname{Spec} R$. Then by the Auslander-Buchsbaum formula,

$$
\operatorname{pd}_{R_{\mathfrak{p}}}\left(R_{\mathfrak{p}} / \mathfrak{p}\right)= \begin{cases}\mathrm{ht}(\mathfrak{p}) & \text { if } \mathfrak{p} \text { is smooth } \\ \infty & \text { otherwise }\end{cases}
$$

This notion was generalized to the noncommutative setting by Brown and Hajarnavis [6]. They define a noetherian (noncommutative) algebra $A$ with prime center $R$ to be homologically homogeneous if the projective dimension of each simple $A$-module $V$ equals the Krull dimension of $R,{ }^{\mathrm{e}}$

$$
\operatorname{pd}_{A}(V)=\operatorname{dim} R=\operatorname{ht}\left(\operatorname{ann}_{R}(V)\right) .
$$

Using this notion, Van den Bergh defines a noncommutative crepant resolution $A$ of a noetherian normal Gorenstein domain $R$ to be a homologically homogeneous endomorphism ring $A=\operatorname{End}_{R}(M)$ of a reflexive finitely generated $R$-module $M$ [9, Definition 4.1].

We propose that if $R$ is a nonnoetherian domain, then homological homogeneity should be replaced by an equality between projective dimension and geometric

e Specifically, if $R$ is a commutative noetherian equidimensional $k$-algebra and $A$ is a module-finite $R$-algebra, then $A$ is homologically homogeneous if all simple $A$-modules have the same projective dimension. 
height, rather than height as in (21). This proposal is illustrated in the following proposition, and is further studied in [4] in the context of homotopy dimer algebras.

Proposition 4.4. Let $A=k Q / I$ be the quiver algebra (19), and set $\mathfrak{m}=x S$. Then

- $A$ is an endomorphism of a reflexive module over its center $Z \cong R$,

$$
A \cong \operatorname{End}_{R}(R \oplus \mathfrak{m}) \cong \operatorname{End}_{Z}\left(A e_{1}\right) .
$$

- Let $V_{i}$ be the simple A-module supported at vertex $i$. Then

$$
\operatorname{pd}_{A}\left(V_{i}\right)=\operatorname{ght}\left(\operatorname{ann}_{e_{i} A e_{i}}\left(V_{i}\right)\right) .
$$

- The smooth locus of $Z$ parametrizes the simple A-module isoclasses of maximal $k$-dimension, and coincides with the open set $U_{S / R} \subset \operatorname{Max} S$.

Therefore, although $A$ and $Z$ are nonnoetherian and $A$ is an infinitely generated $Z$-module, $A$ nevertheless may be viewed as a noncommutative desingularization of its center.

Proof. For the following, denote $\operatorname{Hom}_{R}(-,-)$ by $(-,-)$. Using the labeling of arrows given by the impression (20), we find

$$
\begin{aligned}
\operatorname{End}_{Z}\left(A e_{1}\right) & =\operatorname{End}_{Z}\left(e_{1} A e_{1} \oplus e_{2} A e_{1}\right) \\
& \cong \operatorname{End}_{R}(R \oplus \mathfrak{m}) \\
& \cong\left(\begin{array}{cc}
(R, R) & (\mathfrak{m}, R) \\
(R, \mathfrak{m}) & (\mathfrak{m}, \mathfrak{m})
\end{array}\right) \\
& \cong\left(\begin{array}{ll}
R & S \\
\mathfrak{m} & S
\end{array}\right) \\
& \cong\left(\begin{array}{ll}
e_{1} A e_{1} & e_{1} A e_{2} \\
e_{2} A e_{1} & e_{2} A e_{2}
\end{array}\right) \\
& \cong A .
\end{aligned}
$$

Furthermore, $R \oplus \mathfrak{m}$ is a reflexive $R$-module:

$$
((R \oplus \mathfrak{m}, R), R) \cong(R \oplus S, R) \cong R \oplus \mathfrak{m} .
$$

The minimal projective resolution of $V_{1}$ is

$$
0 \rightarrow\left(\begin{array}{l}
S \\
S
\end{array}\right) \stackrel{\cdot x}{\rightarrow}\left(\begin{array}{l}
R \\
\mathfrak{m}
\end{array}\right) \rightarrow\left(\begin{array}{c}
R / \mathfrak{m} \\
0
\end{array}\right) \cong\left(\begin{array}{l}
k \\
0
\end{array}\right)=V_{1} \rightarrow 0 .
$$

Set $\mathfrak{n}:=(x, y) S \in \operatorname{Max} S$. Then the minimal projective resolution of $V_{2}$ is

$$
0 \rightarrow\left(\begin{array}{l}
S \\
S
\end{array}\right) \stackrel{\cdot(x y-x)}{\longrightarrow}\left(\begin{array}{ll}
R & S \\
\mathfrak{m} & S
\end{array}\right) \stackrel{\cdot\left(\begin{array}{l}
1 \\
y
\end{array}\right)}{\rightarrow}\left(\begin{array}{l}
S \\
S
\end{array}\right) \rightarrow\left(\begin{array}{c}
0 \\
S / \mathfrak{n}
\end{array}\right) \cong\left(\begin{array}{l}
0 \\
k
\end{array}\right)=V_{2} \rightarrow 0
$$

The simple $A$-modules of maximal $k$-dimension are the simples modules with dimension vector $(1,1)$ by $[1$, Lemma 5.1$]$. These modules are parametrized by the 
smooth locus of $\operatorname{Max} Z$, namely $(a b, y) \in k^{*} \times k$, which coincides with $U_{S / R} \subset$ $\operatorname{Max} S$.

Although $A$ is isomorphic to $\operatorname{End}_{Z}\left(A e_{1}\right)$, note that $A$ is not isomorphic to $\operatorname{End}_{Z}\left(A e_{2}\right)$ since

$$
\operatorname{End}_{Z}\left(A e_{2}\right) \cong \operatorname{End}_{R}(S \oplus S) \cong M_{2}(S) .
$$

Furthermore, the moduli space of $\theta$-stable $A$-modules of dimension vector $(1,1)$, for generic stability parameter $\theta$, is precisely the desingularization $\operatorname{Max} S$. (Max $S$ is not a resolution of $\operatorname{Max} R$ since the morphism $\kappa: \operatorname{Max} S \rightarrow \operatorname{Max} R$ is not proper.)

The following example demonstrates the necessity of the assumption $U_{S / R}^{*} \subseteq$ $U_{S / R}$ in Theorem 4.1 .

Example 4.5. Consider the quiver algebra

$$
A=k Q /\left\langle y b a-b a y, y^{2}-b a\right\rangle
$$

with quiver given in Fig. 1, as in Example 4.3. $A$ admits an impression $(\tau, B=k[x])$ where $\tau\left(e_{i}\right)=E_{i i}$ for $i=1,2$, and

$$
\bar{\tau}(a)=\bar{\tau}(b)=\bar{\tau}(y)=x .
$$

By (17), the center $Z$ of $A$ is isomorphic to

$$
R=k\left[x^{2}, x^{3}\right] .
$$

Therefore $Z$ is noetherian. But

$$
S=k[x] \neq R .
$$

However, Theorem 4.1.2 is not applicable to this example because

$$
U_{S / R}^{*}=\mathbb{A}^{1} \not \mathbb{A}^{1} \backslash\{0\}=U_{S / R}
$$

see Remark 2.7.

\section{Acknowledgments}

The author would like to thank Paul Smith and Travis Kopp for useful discussions, and an anonymous referee for useful comments. Part of this paper is based on work supported by the Simons Foundation while the author was a postdoc at the Simons Center for Geometry and Physics at Stony Brook University. His work was also supported in part by a PFGW Grant, which he gratefully acknowledges.

\section{References}

[1] C. Beil, On the noncommutative geometry of square superpotential algebras, J. Algebra 371 (2012) 207-249.

[2] C. Beil, Morita equivalences and Azumaya loci from Higgsing dimer algebras, preprint (2013), arXiv:1301.7059. 
[3] C. Beil, Nonlocality and the central geometry of dimer algebras, in preparation.

[4] C. Beil, Smooth noncommutative blowups of dimer algebras and isolated nonnoetherian singularities, in preparation.

[5] C. Beil, The Bell states in noncommutative algebraic geometry, to appear in Int. J. Quantum Inform.

[6] K. Brown and C. Hajarnavis, Homologically homogeneous rings, Trans. Amer. Math. Soc. 281(1) (1984) 197-208.

[7] G. Leuschke, Non-commutative crepant resolutions: Scenes from categorical geometry, in Progress in Commutative Algebra 1 (De Gruyter, 2012), pp. 293-361.

[8] K. Schwede, Gluing schemes and a scheme without closed points, in Recent Progress in Arithmetic and Algebraic Geometry, AMS Contemporary Mathematics Series (American Mathematical Society, 2005).

[9] M. Van den Bergh, Non-commutative crepant resolutions, in The Legacy of Hendrik Abel (Springer, 2002), pp. 749-770. 